ARTICLE

\title{
Excessive ammonium assimilation by plastidic glutamine synthetase causes ammonium toxicity in Arabidopsis thaliana
}

Takushi Hachiya (1) 1,2,3凶 , Jun Inaba4 ${ }^{4}$, Mayumi Wakazaki ${ }^{4}$, Mayuko Sato (1) ${ }^{4}$, Kiminori Toyooka (i) ${ }^{4}$, Atsuko Miyagi ${ }^{5}$, Maki Kawai-Yamada ${ }^{5}$, Daisuke Sugiura², Tsuyoshi Nakagawa', Takatoshi Kiba (2,4, Alain Gojon ${ }^{6}$ \& Hitoshi Sakakibara (i) 2,4

Plants use nitrate, ammonium, and organic nitrogen in the soil as nitrogen sources. Since the elevated $\mathrm{CO}_{2}$ environment predicted for the near future will reduce nitrate utilization by $\mathrm{C}_{3}$ species, ammonium is attracting great interest. However, abundant ammonium nutrition impairs growth, i.e., ammonium toxicity, the primary cause of which remains to be determined. Here, we show that ammonium assimilation by GLUTAMINE SYNTHETASE 2 (GLN2) localized in the plastid rather than ammonium accumulation is a primary cause for toxicity, which challenges the textbook knowledge. With exposure to toxic levels of ammonium, the shoot GLN2 reaction produced an abundance of protons within cells, thereby elevating shoot acidity and stimulating expression of acidic stress-responsive genes. Application of an alkaline ammonia solution to the ammonium medium efficiently alleviated the ammonium toxicity with a concomitant reduction in shoot acidity. Consequently, we conclude that a primary cause of ammonium toxicity is acidic stress.

\footnotetext{
${ }^{1}$ Department of Molecular and Functional Genomics, Interdisciplinary Center for Science Research, Shimane University, Matsue, Japan. ${ }^{2}$ Graduate School of Bioagricultural Sciences, Nagoya University, Nagoya, Aichi, Japan. ${ }^{3}$ Institute for Advanced Research, Nagoya University, Nagoya, Aichi, Japan. ${ }^{4}$ RIKEN Center for Sustainable Resource Science, Yokohama, Kanagawa, Japan. ${ }^{5}$ Graduate School of Science and Engineering, Saitama University, Saitama, Saitama,

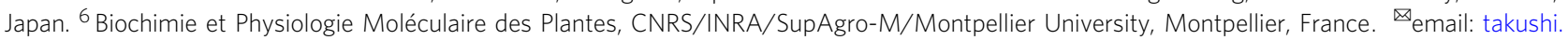
hachiya@life.shimane-u.ac.jp
} 
$\mathrm{N}$ itrate and ammonium are the main sources of nitrogen (N) for most plants. Recent studies suggest that elevated $\mathrm{CO}_{2}$ reduces nitrate reduction in $\mathrm{C}_{3}$ species, such as wheat and Arabidopsis, whereas ammonium utilization does not decrease $^{1}$. One estimate predicts that only a $1 \%$ drop in nitrogen use efficiency could increase worldwide cultivation costs for crops by about $\$ 1$ billion annually ${ }^{2}$. Therefore, increasing ammonium use by crops is an important goal for agriculture as $\mathrm{CO}_{2}$ levels rise in the world; however, millimolar concentrations of ammonium as the sole $\mathrm{N}$ source causes growth suppression and chlorosis in plants, compared with nitrate or lower concentrations of ammonium $^{3-5}$. This phenomenon is widely known as ammonium toxicity, but the primary cause of impaired growth remains to be identified; in the present study, ammonium toxicity is defined as shoot growth suppression with toxic levels of ammonium as the sole $\mathrm{N}$ source compared with nitrate.

Plants grown in high ammonium conditions show several distinct characteristics from those grown in nitrate ${ }^{3-5}$. These toxic symptoms have evoked several hypotheses about the toxic causes, including futile transmembrane ammonium cycling, deficiencies in inorganic cations and organic acids, impaired hormonal homeostasis, disordered $\mathrm{pH}$ regulation, and the uncoupling of photophosphorylation; however, some of the symptoms are not directly associated with growth suppression by ammonium toxicity ${ }^{6}$, making it difficult to determine the toxic cause. Several efforts have isolated ammonium-sensitive mutants in Arabidopsis thaliana and determined their causative genes ${ }^{4,5}$. GMP1 is a causal gene whose deficiency causes stunted growth of primary roots under high ammonium conditions ${ }^{7}$. Given that GMP1 is crucial for synthesizing GDP-mannose as a substrate for $\mathrm{N}$-glycosylation, a lack of $\mathrm{N}$-glycoproteins could be involved in ammonium hypersensitivity. In accordance with this hypothesis, the ammonium-dependent inhibition of primary root growth was shown to be partly attenuated by the lack of a GDP-mannose pyrophosphohydrolase that hydrolyzes GDP-mannose to mannose 1-phosphate and $\mathrm{GMP}^{8}$. In another study, a genetic screen focusing on severely chlorotic Arabidopsis leaves identified AMOS1, a gene encoding a plastid metalloprotease, as a factor for improving ammonium tolerance ${ }^{9}$. Transcriptome analysis revealed that an AMOS1-dependent mechanism regulates more than half of the transcriptional changes triggered by toxic levels of ammonium. On the other hand, recent studies found that ammonium toxicity was partly alleviated by deficiencies in EIN2 and EIN3, regulators of ethylene responses, or by the application of ethylene biosynthesis and action inhibitors ${ }^{10,11}$. This suggests that ammonium toxicity would be mediated via the ethylene signaling pathway.

The above-described genetic studies have succeeded in determining molecular components closely associated with ammonium toxicity. Nevertheless, the initial event that triggers ammonium toxicity remains to be identified and characterized. To address this question, we screened ammonium-insensitive Arabidopsis lines that were expected to attenuate the toxicity and isolated ammonium-insensitive 2 (ami2). Interestingly, the defect in ami2 was downregulation of the GLUTAMINE SYNTHETASE 2 (GLN2) gene encoding an ammonium assimilatory enzyme localized in the plastid. We identified that in the presence of toxic levels of ammonium, large levels of proton production, due to excessive assimilation of ammonium by GLN2, aggravate the acidic burden and lead to plant toxicity.

\section{Results}

A genetic screen isolated an ammonium-insensitive mutant. To find ammonium-insensitive lines, a gain-of-function population of the Arabidopsis FOX (full-length cDNA overexpressing) lines ${ }^{12}$ was used. An apparent ammonium-insensitive mutant was identified that shows enhanced growth of cotyledons that are greener than wild-type $(\mathrm{Col})$ when grown on $10 \mathrm{mM}$ ammonium in the form of $5 \mathrm{mM}\left(\mathrm{NH}_{4}\right)_{2} \mathrm{SO}_{4}$ as the sole $\mathrm{N}$ source; the mutant was named ami2 (Fig. 1a). The fresh weights of ami2 11-d-old shoots were approximately double those of $\mathrm{Col}$ when grown on ammonium (Fig. 1b). A similar growth enhancement in ami2 was observed in the media containing $10 \mathrm{mM} \mathrm{NH}_{4} \mathrm{Cl}$ as the sole $\mathrm{N}$ source (Supplementary Fig. 1a, b), confirming that ami2 is tolerant to ammonium. In the following experiments, we used $5 \mathrm{mM}$ $\left(\mathrm{NH}_{4}\right)_{2} \mathrm{SO}_{4}$ as the ammonium source. In contrast, in media containing $10 \mathrm{mM}$ nitrate or $5 \mathrm{mM}$ ammonium plus $5 \mathrm{mM}$ nitrate, the shoot fresh weights of ami2 were less than those of Col. In media containing $10 \mathrm{mM}$ ammonium, the percentage increase in fresh weight of ami2 relative to Col was much larger for shoots (by ca. 110\%) than for roots (by ca. 50\%) (Fig. 1c). The greater shoot growth in ami2 was reduced in media with lower concentrations of ammonium $(0.4,2 \mathrm{mM})$, in which the shoot growth of Col was greater than that when grown on media containing $10 \mathrm{mM}$ ammonium (Fig. 1d). Moreover, nitrate addition in the presence of $10 \mathrm{mM}$ ammonium attenuated the deficiency in shoot growth more effectively in Col than in ami2, decreasing the growth difference in a concentration-dependent
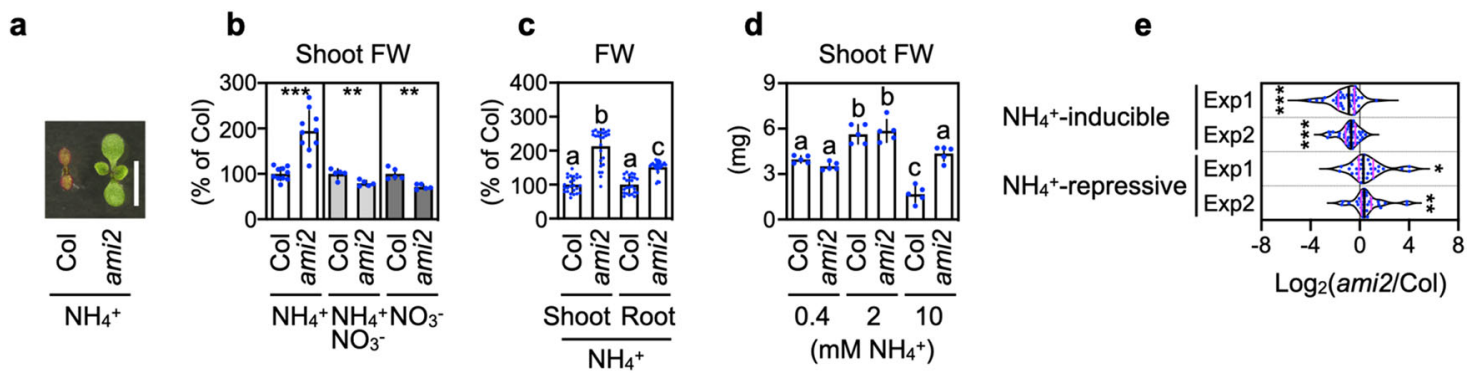

Fig. 1 Enhanced shoot growth of ami2 in the presence of $\mathbf{1 0} \mathbf{~ m M}$ ammonium. a A representative photograph of shoots from the 11-d-old wild-type (Col) and ami2 grown on media containing $10 \mathrm{mM}$ ammonium. The scale bar represents $5 \mathrm{~mm}$. b Fresh weights (FW) of shoots from $11-\mathrm{d}$-old Col and ami2 grown on media containing $10 \mathrm{mM}$ ammonium (mean $\pm \mathrm{SD} ; n=10), 5 \mathrm{mM}$ ammonium nitrate ( $\mathrm{mean} \pm \mathrm{SD} ; n=5$ ), or $10 \mathrm{mM}$ nitrate (mean $\pm \mathrm{SD} ; n=5$ ). $\mathbf{c}$ FW of shoots and roots from 11-d-old Col and ami2 grown on media containing $10 \mathrm{mM}$ ammonium (mean \pm SD; $n=26$ ). d FW of 11 -d-old Col and ami2 shoots grown on media containing $0.4,2$, or $10 \mathrm{mM}$ ammonium (mean $\pm \mathrm{SD}, n=5$ ). e Violin plots of the differences in the expression of the ammonium stressresponsive genes between the 10 - $d$-old $\mathrm{Col}$ and ami2 shoots $3 \mathrm{~d}$ after transfer to media containing $10 \mathrm{mM}$ ammonium. The gene list was obtained from Li et al. $^{9}$ (for further details, see Supplementary Data 1). Two independent experiments (Exp1 and Exp2) were performed. Nine shoots from three plates constituted a single biological replicate. An individual violin plot shows the median (black line) and the 25th to 75th percentiles (magenta line). $\mathbf{b}$, $\mathbf{d}$ Six shoots from one plate constituted a single biological replicate. b, e ${ }^{\star} P<0.05$; ${ }^{\star \star} P<0.01 ;{ }^{\star \star} P<0.001$ (Welch's $t$-test). $\mathbf{c}$, d Different letters denote significant differences at $P<0.05$ (Tukey-Kramer's multiple comparison test). 
manner (Supplementary Fig. 1c, d). A time-course analysis of shoot growth revealed that increased ammonium tolerance of the ami2 plants compared to Col was significant as soon as $5 \mathrm{~d}$ after culture initiation (Supplementary Fig. 1e). These results indicate that ammonium tolerance in ami2 shoots is manifested specifically under harsh ammonium conditions.

To corroborate this enhanced ammonium tolerance in ami2, we performed microarray experiments and compared the expression of genes responsive to toxic levels of ammonium ${ }^{9}$ between the Col and ami2 shoots growing in media containing $10 \mathrm{mM}$ ammonium (Fig. 1e and Supplementary Data 1). The transcript levels of ammonium-inducible genes were significantly reduced in ami2 shoots compared with Col shoots, whereas those of ammonium-repressive genes showed the opposite trend. A reverse transcription-quantitative PCR (RT-qPCR) analysis confirmed that expression of CMCU, MIOX2, and $\mathrm{PDH} 2$, ammonium-inducible genes 9,13 , was upregulated in Col shoots under $10 \mathrm{mM}$ ammonium compared with $10 \mathrm{mM}$ nitrate, and this ammonium induction was significantly suppressed in ami2 shoots (Supplementary Fig. 2a). We confirmed that the expression of a housekeeping gene TIP41 was little changed, and that of nitrate-inducible marker gene $N I R^{14}$ was increased under $10 \mathrm{mM}$ nitrate condition (Supplementary Fig. 2b, c). Collectively, these results indicate that ammonium toxicity is attenuated in ami2 shoots.

GLUTAMINE SYNTHETASE 2 is a causative gene for ammonium toxicity. Next, to identify the causative gene in ami2, we recovered the transgene in a vector using specific primers and sequenced the construct. The gene was identified as GLUTAMINE SYNTHETASE 2 (GLN2), the sole plastidic isoform in A. thaliana (Fig. 2a and Supplementary Fig. 3a). Because the transgene was driven by the cauliflower mosaic virus $35 \mathrm{~S}$ promoter, we expected that overexpression of GLN2 would enhance ammonium tolerance; however, in media containing $10 \mathrm{mM}$ ammonium, the transcript levels of GLN2 in ami2 shoots were downregulated to about $5 \%$ of those in Col (Fig. 2b). In contrast, among the major cytosolic GLUTAMINE SYNTHETASE genes (GLN1s), GLN1;1 was upregulated in ami2 shoots, but GLN1;2 and GLN1;3 were slightly downregulated (Supplementary Fig. 3b). Also, an immunoblot analysis using anti-GLN antibodies ${ }^{15}$ confirmed that the protein levels of GLN2 were remarkably lower in ami2 shoots compared with Col, whereas the signal intensities corresponding to GLN1s were comparable between the mutant and wild type (Fig. 2c). These findings suggested that overexpression of GLN2 cDNA would result in a cosuppression event ${ }^{16}$, which makes it difficult to test for phenotypic complementation by introducing the GLN2 transgene. To ensure that reduced expression of GLN2 enhances ammonium tolerance, we obtained another GLN2-deficient line having a T-DNA insertion at the $3^{\prime}$-UTR region of GLN2 (SALK_051953, designated as $g \ln 2$, Supplementary Fig. 3a). As expected, $g \ln 2$ phenocopied ami2 in terms of the reduced GLN2 and GLN2 expression (Fig. 2b, c), the enhanced ammonium tolerance (Fig. 2d, e and Supplementary Fig. 3c, d), and the lowered induction of ammonium-inducible genes when grown on ammonium (Supplementary Fig. 2a). Furthermore, a similar ammonium tolerance was observed in the GLN2-knockout line (SALK_071292, designated as gln2-2, Supplementary Fig. 3a, e, f) which was recently isolated in another research group ${ }^{17}$. Thus, we concluded that GLN2 is a causative gene for ammonium toxicity.

Shoot GLN2 causes while root GLN1;2 attenuates ammonium toxicity. Previous studies had reported that mutants deficient in AtGLN1;2 were hypersensitive to millimolar concentrations of a

b
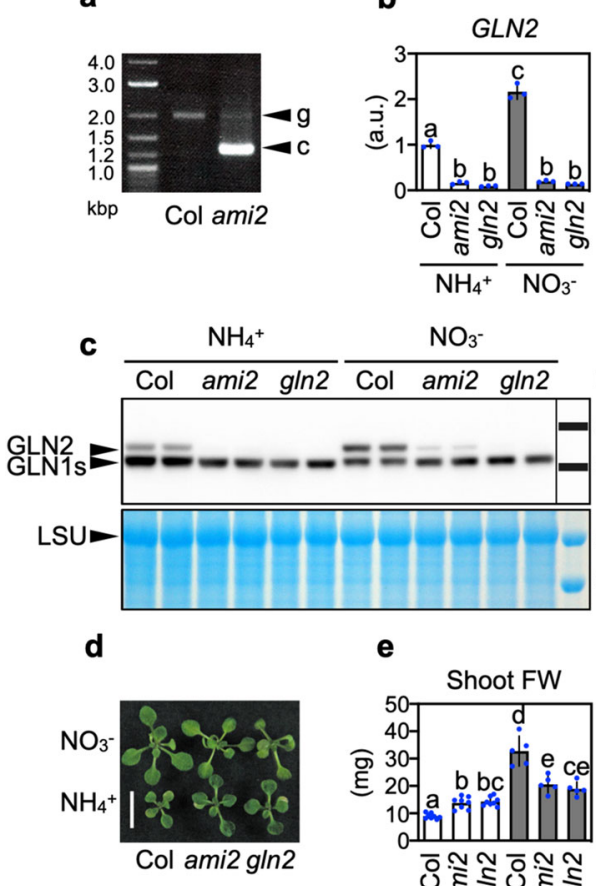

$\mathrm{NO}_{3}-$

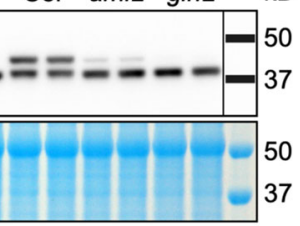

e

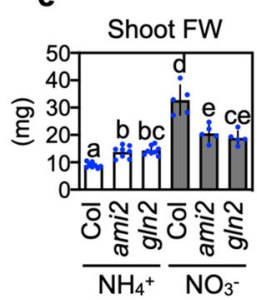

Fig. 2 Downregulation of GLN2 enhances ammonium tolerance. a Genomic PCR using GLN2-specific primers. g and c denote the PCR fragments derived from genomic DNA and cDNA sequences corresponding to GLN2, respectively. b Relative transcript levels of GLN2 in the shoots of 10-d-old Col, ami2, and gln2 $3 \mathrm{~d}$ after transfer to media containing $10 \mathrm{mM}$ ammonium or $10 \mathrm{mM}$ nitrate (mean $\pm \mathrm{SD} ; n=3$ ). Six shoots from two plates constituted a single biological replicate. c Immunodetection of GLN1s and GLN2 isoproteins using specific antisera following SDS-PAGE and immunoblotting of total proteins from the shoots of 12-d-old Col, ami2, and gln2 $5 \mathrm{~d}$ after transfer to media containing $10 \mathrm{mM}$ ammonium or $10 \mathrm{mM}$ nitrate. LSU denotes large subunits of RuBisCO. $\mathbf{d}$ A representative photograph of shoots from 14-d-old Col, ami2, and gln2 $7 \mathrm{~d}$ after transfer to media containing $10 \mathrm{mM}$ ammonium or $10 \mathrm{mM}$ nitrate. The scale bar represents $10 \mathrm{~mm}$. e FW of shoots from 14-d-old Col, ami2, and gln2 $7 \mathrm{~d}$ after transfer to media containing $10 \mathrm{mM}$ ammonium (mean $\pm \mathrm{SD} ; n=8$ ) or $10 \mathrm{mM}$ nitrate (mean $\pm S D ; n=5$ ). Mean values of three shoots from one plate constituted a single biological replicate. b, e Different letters denote significant differences at $P<0.05$ (Tukey-Kramer's multiple comparison test).

ammonium ${ }^{18-20}$. We also confirmed the ammonium hypersensitivity of $g \ln 1 ; 2-1$ and $g \ln 1 ; 2-2$ (Supplementary Fig. 4a-d). GLN2 and GLN1;2, therefore, have opposite effects on ammonium toxicity. To discover how GLN2 and GLN1;2 are involved in the toxicity, we evaluated the distribution of GLN2 and GLN1;2 expressions between shoots and roots in Col plants. In the presence of ammonium or nitrate, the steady-state levels of GLN2 expression were consistently higher in the shoots than in the roots, whereas expression of GLN1;2 was much higher in the roots (Fig. 3a, b and Supplementary Fig. 5a, b), implying that both shoot GLN2 and root GLN1;2 could affect ammonium toxicity. To support this hypothesis, we performed a growth analysis using reciprocally grafted plants between $\mathrm{Col}$ and ami2 (Fig. $3 \mathrm{c}$ and Supplementary Fig. 5c) and between Col and gln1;2-1 (Fig. 3d and Supplementary Fig. 5d). Prior to the analysis, we confirmed that shoot expression of GLN2 was lower in the ami2-derived shoots irrespective of root-genotype (Supplementary Fig. 6) because GLN2 mRNA is suggested to be root-to-shoot mobile ${ }^{21}$. Only 

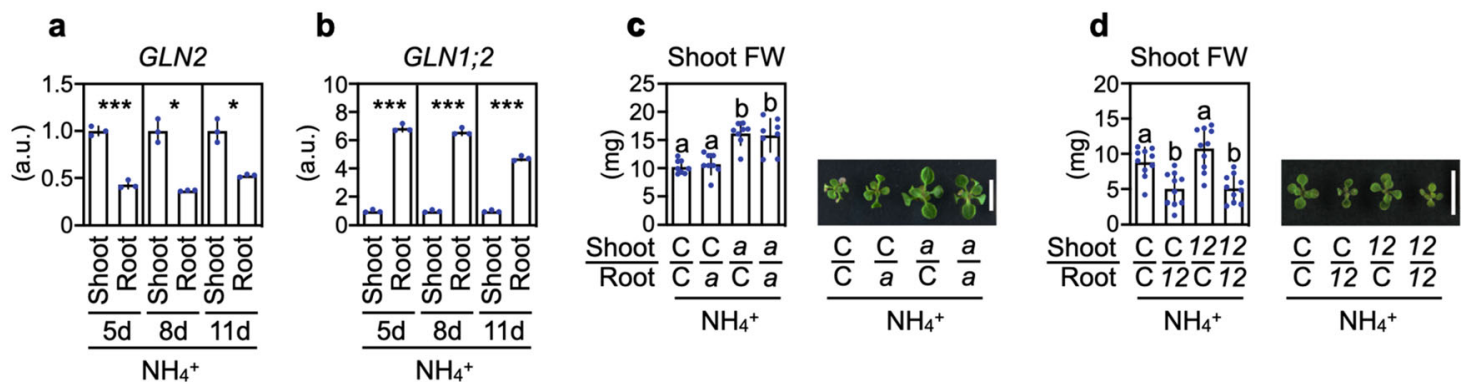

Fig. 3 Shoot GLN2 causes ammonium toxicity, whilst root GLN1;2 attenuates it. a Relative transcript levels of GLN2 in the shoots and roots of 5-, 8-, or 11$\mathrm{d}$-old Col grown on media containing $10 \mathrm{mM}$ ammonium (mean $\pm \mathrm{SD} ; n=3$ ). b Relative transcript levels of GLN1;2 in the shoots and roots of 5-, 8-, or 11-dold Col grown on media containing $10 \mathrm{mM}$ ammonium (mean $\pm S D ; n=3$ ). a, b Twelve shoots and roots from one plate constituted a single biological replicate. ${ }^{\star} P<0.05 ;{ }^{\star \star} P<0.01$; ${ }^{\star \star \star} P<0.001$ (Welch's $t$-test). c FW of shoots from 17-d-old reciprocally grafted plants between Col (C) and ami2 (a) $7 \mathrm{~d}$ after transfer to media containing $10 \mathrm{mM}$ ammonium (mean $\pm \mathrm{SD} ; n=8$ ). d FW of shoots from 17-d-old reciprocally grafted plants between Col (C) and gln1;2-1 $7 \mathrm{~d}$ after transfer to media containing $10 \mathrm{mM}$ ammonium (mean $\pm \mathrm{SD} ; n=10$ ). c, d One shoot from one plate constituted a single biological replicate. Different letters denote significant differences at $P<0.05$ (Tukey-Kramer's multiple comparison test). Representative photograph of 17-d-old shoots $7 \mathrm{~d}$ after transfer to media containing $10 \mathrm{mM}$ ammonium are shown. The scale bar represents $10 \mathrm{~mm}$.

when the scion was derived from ami2 was shoot growth significantly enhanced in the presence of $10 \mathrm{mM}$ ammonium (Fig. 3c). On the other hand, deficiency in root GLN1;2 content was sufficient to decrease shoot growth in ammonium (Fig. 3d). Further, we observed that in ammonium-grown plants, the total enzymatic activities of GLNs were significantly reduced by ca. $30-40 \%$ in 5 -d-old shoots of ami2 and $g \ln 2$ and by ca. $40-60 \%$ in 5 -d-old roots of $g \ln 1 ; 2-1$ and $g \ln 1 ; 2-2$ compared with Col (Supplementary Fig. 7a, b). Additionally, partially compensatory inductions of other GLNs were found in the mutants (Supplementary Fig. 7c, d). Our findings demonstrate that although shoot GLN2 causes ammonium toxicity in the shoot, root GLN1;2 attenuates ammonium toxicity.

Lowered GLN2 activity reduces the conversion of ammonium to amino acids. It is generally held that ammonium per se is a toxic compound ${ }^{22}$. On the other hand, a deficiency in GLN2 content should lead to ammonium accumulation in the shoot. Our determination of shoot ammonium content revealed that ami2 and $g \ln 2$ shoots grown on $10 \mathrm{mM}$ ammonium both accumulated more than $100 \mu \mathrm{mol} \mathrm{g}^{-1}$ fresh weight of ammonium (Fig. 4a), albeit the two mutants accumulated more fresh weight than Col (Supplementary Fig. 8). In Col shoots, the ammonium concentrations were $12.9 \pm 0.9 \mu \mathrm{mol} \mathrm{g}^{-1}$ and $2.2 \pm 0.4 \mu \mathrm{mol} \mathrm{g}$ (mean $\pm \mathrm{SD}$ ) under $10 \mathrm{mM}$ ammonium and $10 \mathrm{mM}$ nitrate, respectively (Fig. 4a). This result indicates that ammonium assimilation by GLN2, rather than ammonium accumulation, triggers ammonium toxicity in the shoot.

An ample supply of ammonium increases the concentrations of amino acids compared with nitrate supply alone $e^{6,23}$. In particular, the molar ratios of Gln to Glu are elevated at higher ammonium levels, suggesting that Gln synthesis by glutamine synthetase (GLN) overflows glutamate synthase (GOGAT) capacity. Our hierarchical cluster analysis of amino acid content in shoots clearly demonstrated that the type of $\mathrm{N}$ source, i.e., $10 \mathrm{mM}$ ammonium or nitrate, was the strongest determinant for plant amino acid composition (Fig. 4b and Supplementary Fig. 9a). In this analysis, Col and the GLN2-deficient lines are categorized into separate clusters depending on the $\mathrm{N}$ source. The molar ratio of Gln to Glu (Fig. 4c), total amino acid-N content per amino acid (Supplementary Fig. 9b), total amino acid-N content per fresh weight (Supplementary Fig. 9c), and the molar ratios of $\mathrm{N}$ to $\mathrm{C}$ in total amino acids (Supplementary Fig. 9d) were consistently larger in ammonium-grown shoots than nitrate-grown shoots, and this large ammonium- $\mathrm{N}$ input was partly but significantly attenuated by GLN2 deficiency. These findings suggest that the GLN2 reaction leads to excessive incorporation of ammonium-N into amino acids in shoots when toxic levels of ammonium are present. In addition, we observed that total $\mathrm{N}$ and protein concentrations were generally larger in ammonium-grown shoots than nitrate-grown shoots (Supplementary Fig. 10a, b). In ammonium-grown shoots, the total $\mathrm{N}$ concentrations were significantly reduced by GLN2 deficiency (Supplementary Fig. 10a), and the protein concentrations were marginally decreased (Supplementary Fig. 10b). Collectively, the shoot GLN2 would facilitates the accumulation of assimilated $\mathrm{N}$ in the shoot under toxic ammonium condition.

Ammonium assimilation by GLN2 causes acidic stress. The amino acid profiles suggested that metabolic imbalances due to excessive ammonium assimilation by GLN2 could be a cause of ammonium toxicity. This hypothesis is supported by the observation that shoot growth of Col was much lower under $10 \mathrm{mM}$ ammonium than under $2 \mathrm{mM}$ ammonium and the shoot growth enhancement by GLN2 deficiency was determined only under 10 $\mathrm{mM}$ ammonium as already explained above. We have previously demonstrated that nitrate addition at adequate concentrations mitigates ammonium toxicity without reducing amino acid accumulation $^{6}$. Therefore, a phenomenon triggered by some GLN2-mediated process other than amino acid accumulation should be a cause of ammonium toxicity. Notably, the GLN reaction is a proton-producing process ${ }^{24}$. The stoichiometry of this reaction is two protons per each glutamine produced, one proton of which is derived from ATP hydrolysis and the other is from deprotonation of $\mathrm{NH}_{4}{ }^{+}$. Conversely, the subsequent ferredoxin-dependent glutamate synthase (Fd-GOGAT) reaction consumes two protons per one glutamine incorporated. Given that the molar ratio of Gln to Glu was about 10 in the ammonium condition but close to 1 in the nitrate condition (Fig. 4c), proton production in the ammonium condition could proceed beyond its consumption. Strikingly, a previous study found that $43 \%$ of ammonium-inducible genes correspond to acidic stress-inducible genes in Arabidopsis roots ${ }^{13,25}$. We found that ammoniuminducible genes whose expression was reduced by GLN2 deficiency under $10 \mathrm{mM}$ ammonium condition (Supplementary Fig. 2a) are known as acid-inducible genes ${ }^{25}$. Thus, we hypothesized that excessive ammonium assimilation by GLN2 causes acidic stress to the plants growing in ammonium.

We re-surveyed our microarray data by focusing on previously identified acidic stress-responsive genes $^{25}$ (Fig. $5 \mathrm{a}$ and 

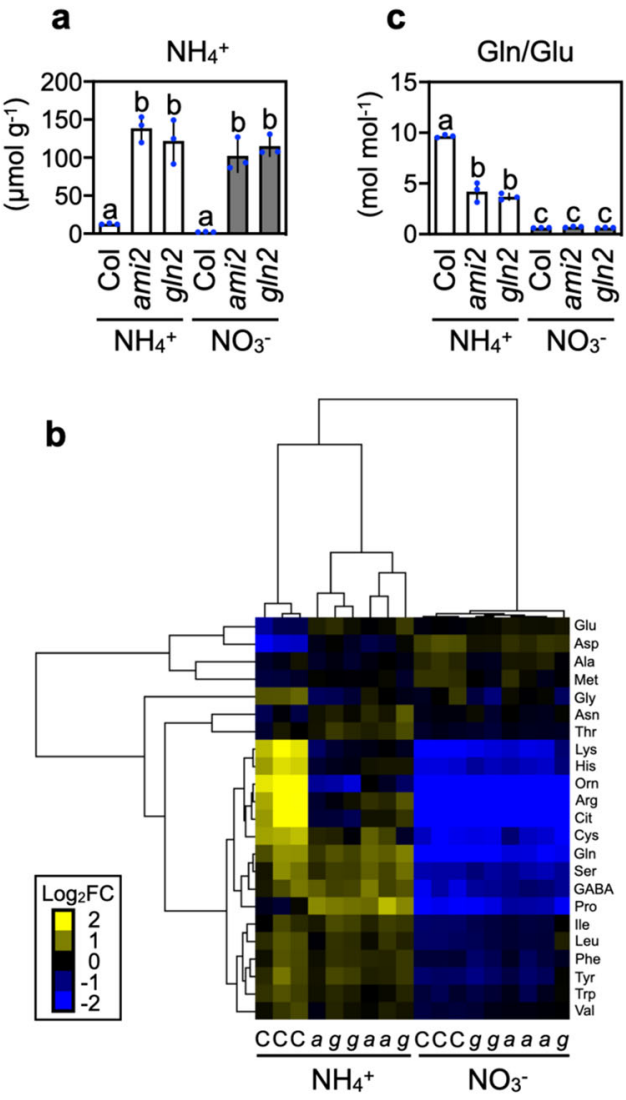

Fig. 4 Lowered activity of GLN2 reduces the conversion of ammonium to amino acids. a The shoot ammonium content of 12 -d-old $\mathrm{Col}$, ami2, and gln2 $5 \mathrm{~d}$ after transfer to media containing $10 \mathrm{mM}$ ammonium or $10 \mathrm{mM}$ nitrate (mean $\pm \mathrm{SD} ; n=3$ ). Three shoots from one plate constituted a single biological replicate. $\mathbf{b}$ Hierarchical clustering of the shoot amino acid content of 12-d-old Col (C), ami2 (a), and gln2 ( $g$ ) $5 \mathrm{~d}$ after transfer to media containing $10 \mathrm{mM}$ ammonium or $10 \mathrm{mM}$ nitrate. The color spectrum from yellow to blue corresponds to the relative content of each amino acid. The fold-change (FC) value on each amino acid was calculated by dividing the amino acid content of a sample by the mean content of all 18 samples. $\log _{2} \mathrm{FC}$ denotes the base- 2 logarithm of FC. c The molar ratio of Gln to Glu in the shoots of 12-d-old Col, ami2, and gln2 $5 \mathrm{~d}$ after transfer to media containing $10 \mathrm{mM}$ ammonium or $10 \mathrm{mM}$ nitrate (mean $\pm \mathrm{SD} ; n=3$ ). $\mathbf{a}, \mathbf{c}$ Different letters denote significant differences at $P<0.05$ (Tukey-Kramer's multiple comparison test). b, c Six shoots from two plates constituted a single biological replicate. Three biological replicates were sampled separately three times.

Supplementary Data 2). All acidic stress-inducible genes were entirely downregulated in ami2 shoots compared with Col, whereas the acidic stress-repressive genes showed the opposite trend. The transcript levels of ALMT1, a typical acidic stressinducible gene, were determined in the shoots of $\mathrm{Col}$ and ami2 plants incubated in $10 \mathrm{mM}$ ammonium or nitrate with or without methionine sulfoximine (MSX), an inhibitor of the GLN reaction (Fig. 5b). ALMT1 expression was much higher in the ammoniumtreated Col shoots than in the nitrate-treated samples. This ammonium-dependent induction was significantly diminished in the ami2 shoots and was mimicked by MSX treatment. Also, other proton-inducible genes such as GABA-T, GAD1, GDH2, $P G I P 1$, and $P G I P 2^{26}$ were ammonium-inducible, and their inductions were suppressed or attenuated by GLN2 deficiency (Supplementary Fig. 11a). GAD1 catalyzes the conversion from Glu to GABA with consumption of proton, acting as a $\mathrm{pH}$ regulating pathway ${ }^{26}$. The molar ratios of GABA to Glu were larger in ammonium-grown shoots than nitrate-grown shoots, and these larger ratios were significantly reduced by GLN2 deficiency (Supplementary Fig. 11b), implying facilitated proton consumption by GAD1 in a GLN2-dependent manner under ammonium condition. These results support our hypothesis associating ammonium assimilation with acidic stress. Moreover, in Col and ami2 reciprocally grafted plants growing in the ammonium condition, ALMT1 expression was significantly lower in the ami2-derived shoots than the Col-derived shoots (Supplementary Fig. 11c), indicating that shoot GLN2 locally causes acidic stress to the shoot. Furthermore, ALMT1 expression was analyzed using grafted plants between $\mathrm{Col}$ and a mutant lacking the STOP1 transcription factor (stop1-KO) that induces ALMT1 to respond to acidic stress ${ }^{26}$ (Supplementary Fig. 11d). In the stop1-KO-derived shoots, the ammonium-dependent induction of ALMT1 disappeared, reconfirming the notion that acidic stress occurs in plants growing in ammonium.

It is widely accepted that the reduction from nitrate to ammonium consumes a proton, suggesting that nitrate reduction could attenuate acidic stress caused by excess ammonium and might explain why nitrate addition alleviates ammonium toxicity. To verify this hypothesis, we analyzed shoot expression of ALMT1 using grafted plants between Col and the NITRATE REDUCTASE-null mutant (designated as NR-null) ${ }^{14}$ (Supplementary Fig. 11e). The addition of $2.5 \mathrm{mM}$ nitrate diminished the ammonium-dependent ALMT1 induction in the Col-derived shoots but not in the NR-null-derived shoots, thereby supporting the above hypothesis.

To obtain direct evidence for ammonium-dependent proton production, we measured the proton concentrations of water extracts from the $\mathrm{Col}$ and ami2 shoots incubated in media containing $10 \mathrm{mM}$ ammonium or nitrate with or without MSX (Fig. 5c). The ammonium-treated Col shoots contained the highest concentrations of protons; proton content was significantly decreased by GLN2 deficiency and by MSX treatment to levels comparable to those in nitrate-treated shoots. A similar trend was observed among the Col, ami2, and $g \ln 2$ shoots grown on ammonium- or nitrate-containing media (Supplementary Fig. 11f).

The presence of ammonium in cultures generally acidifies the external media ${ }^{13}$. Thus, we quantified the proton efflux from the Col and ami2 shoots incubated in media containing $10 \mathrm{mM}$ ammonium or nitrate with or without MSX (Fig. 5d). Incubation of the Col shoots in the presence of ammonium strongly acidified the external media, which was alleviated by GLN2 deficiency and by MSX treatment. A similar tendency was observed by qualitative measurements with a $\mathrm{pH}$ indicator of proton effluxes from mesophyll cells where GLN2 is predominantly expressed (Supplementary Fig. 11g). Thus, we conclude that ammonium assimilation by GLN2 without nitrate increases shoot acidity.

Ammonium toxicity is closely associated with acidic stress. If acidic stress rather than ammonium accumulation has a dominant effect on ammonium toxicity, an application of alkaline ammonia should reduce the toxicity. Given that the GLN2 reaction is a primary cause of increased acidic stress, an elevation in medium $\mathrm{pH}$ may increase the shoot growth of Col more effectively than that of the GLN2-deficient mutants. As expected, the addition of an ammonia solution to media containing $10 \mathrm{mM}$ ammonium elevated the $\mathrm{pH}$ from 5.7 to 6.7 and significantly improved shoot growth with a concomitant decrease in acidity (Fig. 6a, b). Fresh weights of Col shoots grown at $\mathrm{pH} 6.7$ increased by ca. $180 \%$ compared with those grown at $\mathrm{pH} 5.7$, whereas fresh weights of ami2 and $g \ln 2$ shoots only increased by ca. $30 \%$ and $60 \%$, respectively (Fig. $6 \mathrm{c}$ ). In addition, the acid- 
a

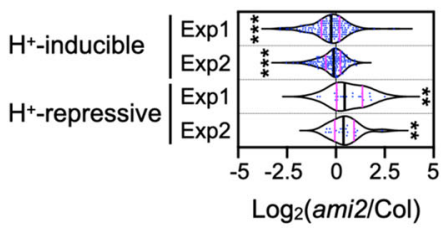

b

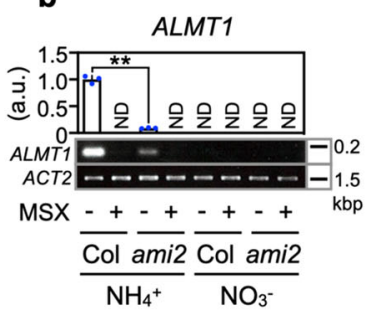

c

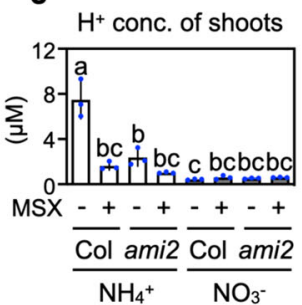

d

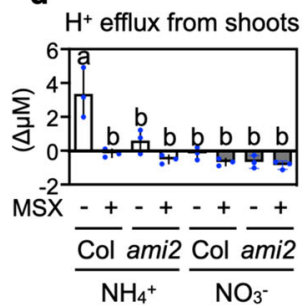

Fig. 5 Ammonium assimilation by GLN2 causes acidic stress. a Violin plots of the differences in expression of the acidic stress-responsive genes between the 10 -d-old $\mathrm{Col}$ and ami2 shoots $3 \mathrm{~d}$ after transfer to media containing $10 \mathrm{mM}$ ammonium. The gene list was obtained from Lager et al. ${ }^{25}$ (For further details, see Supplementary Data 2). Two independent experiments (Exp1 and Exp2) were performed. Nine shoots from three plates constituted a single biological replicate. An individual violin plot shows the median (black line) and the 25th to 75th percentiles (magenta line). $\mathbf{b}$ Effects of MSX treatment on the relative transcript level of $A L M T 1$ in the $10-\mathrm{d}$-old $\mathrm{Col}$ and ami2 shoots $3 \mathrm{~d}$ after transfer to media containing $10 \mathrm{mM}$ ammonium or $10 \mathrm{mM}$ nitrate. The transcript levels were evaluated both by RT-qPCR (mean \pm SD; $n=3$ ) and semi-quantitative RT-PCR with agarose gel electrophoresis. ACTIN2 (ACT2) was the internal standard. Three shoots from one plate constituted a single biological replicate. $\mathbf{c}$ Effects of MSX treatment on proton concentrations in water extracts from the 10-d-old Col and ami2 shoots $3 \mathrm{~d}$ after transfer to media containing $10 \mathrm{mM}$ ammonium or $10 \mathrm{mM}$ nitrate (mean \pm SD; $n=3$ ). Three shoots from one plate constituted a single biological replicate. $\mathbf{d}$ Effects of MSX treatment on proton efflux rates from the 10-d-old Col and ami2 shoots $3 \mathrm{~d}$ after transfer to media containing $10 \mathrm{mM}$ ammonium or $10 \mathrm{mM}$ nitrate (mean $\pm \mathrm{SD} ; n=3$ ). Three shoots from one plate constituted a single biological replicate. $\mathbf{a}, \mathbf{b}{ }^{\star} P<0.05 ;{ }^{\star \star} P<0.01 ;{ }^{\star \star \star} P<0.001$ (Welch's $t$-test). c, $\mathbf{d}$ Different letters denote significant differences at $P<0.05$ (Tukey-Kramer's multiple comparison test).

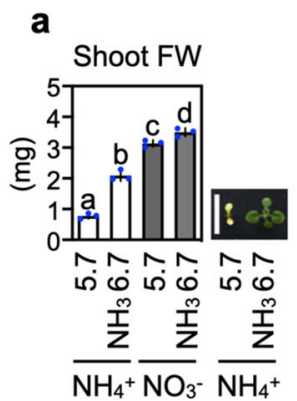

b $\mathrm{H}^{+}$conc. of shoots

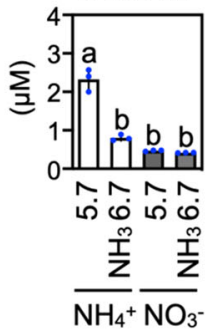

C

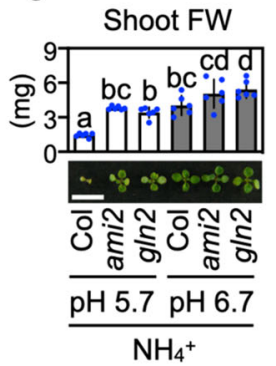

d

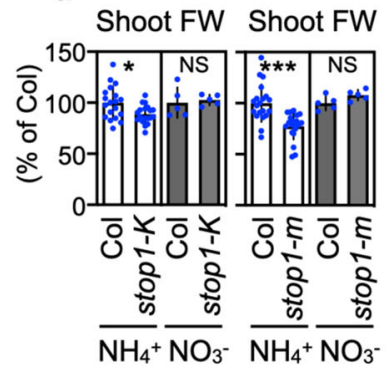

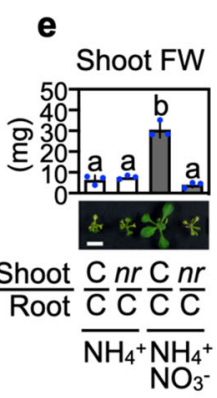

Fig. 6 Ammonium toxicity is closely linked with acidic stress. $\mathbf{a}$, b Effects of $\mathrm{NH}_{3}$ application on shoot $\mathrm{FW}$ and proton concentrations in water extracts of 5 -d-old Col grown on media containing $10 \mathrm{mM}$ ammonium or $10 \mathrm{mM}$ nitrate (mean $\pm \mathrm{SD} ; n=3$ ). Thirty-seven shoots from one plate constituted a single biological replicate. The $\mathrm{pH}$ was adjusted to $\mathrm{pH} 5.7$ with $1 \mathrm{~N} \mathrm{KOH}$; subsequently, a commercial $25 \%(\mathrm{v} / \mathrm{v})$ ammonia solution was added to adjust the $\mathrm{pH}$ from 5.7 to 6.7. A representative photograph of 11-d-old shoots grown on $10 \mathrm{mM}$ ammonium (12 plants per plate) is shown. $\mathbf{c}$ Effects of higher pH on the FW of shoots from 11-d-old Col, ami2, and gln2 grown on media containing $10 \mathrm{mM}$ ammonium for $11 \mathrm{~d}$ (mean $\pm \mathrm{SD} ; n=6$ ). Three shoots from one plate constituted a single biological replicate. The $\mathrm{pH}$ was adjusted to $\mathrm{pH} 5.7$ with $1 \mathrm{~N} \mathrm{KOH}$; subsequently, $1 \mathrm{~N} \mathrm{NaOH}$ was used to adjust the pH from 5.7 to 6.7 to maintain the potassium concentration constant among all samples. A representative photograph of 11-d-old shoots is shown. $\mathbf{d}$ FW of shoots from 11-d-old Col, stop1-KO mutant (stop1-k), and the stop1 mutant (stop1-m) grown on media containing $10 \mathrm{mM}$ ammonium (mean \pm SD; $n=20$ ) or 10 mM nitrate (mean $\pm \mathrm{SD} ; n=5$ ) for $11 \mathrm{~d}$. Six shoots from one plate constituted a single biological replicate. ${ }^{\star} P<0.05$; ${ }^{\star \star} P<0.01$; ${ }^{\star \star \star} P<0.001$ (Welch's $t$-test). e FW of shoots from 17-d-old plants grafted between $\mathrm{Col}(\mathrm{C})$ and the NR-null mutant (nr) $7 \mathrm{~d}$ after transfer to media containing $10 \mathrm{mM}^{2} \mathrm{mmonium}\left(\mathrm{NH}_{4}{ }^{+}\right)$or 2.5 $\mathrm{mM}$ nitrate and $10 \mathrm{mM}$ ammonium $\left(\mathrm{NH}_{4}{ }^{+} \mathrm{NO}_{3}{ }^{-}\right.$) conditions (mean $\pm \mathrm{SD} ; n=3$ ). One shoot from one plate constituted a single biological replicate. $\mathrm{A}$ representative photograph of 17 -d-old shoots $7 \mathrm{~d}$ after transfer to media is shown. a-c, e Different letters denote significant differences at $P<0.05$ (Tukey-Kramer's multiple comparison test). The scale bar represents $10 \mathrm{~mm}$.

sensitive STOP1-deficient mutants had slightly but significantly lower shoot growth when grown in $10 \mathrm{mM}$ ammonium (Fig. 6d), although their acid-hypersensitivity has been described only in roots to date 26,27 . Moreover, the NR-null-derived shoots that lack a proton-consuming nitrate reduction capacity failed to attenuate ammonium toxicity by nitrate addition (Fig. 6e). Collectively, our results lead to the conclusion that acidic stress is one of the primary causes of ammonium toxicity.

GLN2 causes ammonium toxicity independently of NRT1.1. We have already reported that Arabidopsis NITRATE TRANSPORTER 1.1 (NRT1.1), acting as a nitrate transceptor, also aggravates ammonium toxicity ${ }^{28}$. This result was recently confirmed by another group ${ }^{11}$. Thus, we investigated whether NRT1.1 and GLN2 increase the sensitivity to ammonium through a common mechanism. A RT-qPCR analysis revealed that deficiency of either NRT1.1 or GLN2 did not downregulate the expression of the other gene (Supplementary Fig. 12a), and that GLN2 expression was almost 3-times higher in nrt1.1 than in Col. Therefore, the enhanced ammonium tolerance of $n r t 1.1$ cannot be explained by reduced GLN2 expression as in $g \ln 2$. Moreover, a homozygous double mutant of NRT1.1 and GLN2 showed slightly but significantly larger shoot fresh weight, leaf number, shoot diameter, and chlorophyll content compared with any of the single mutants (Supplementary Fig. 12b-d). These findings suggest that NRT1.1 and GLN2 would be implicated in ammoniumsensitivity independently.

\section{Discussion}

Although ammonium is believed to be a toxic compound for plant growth, our results demonstrate that ammonium assimilation by shoot GLN2 rather than ammonium accumulation is a major cause of ammonium toxicity. In plants growing in toxic levels of ammonium as a sole $\mathrm{N}$ source, assimilation of 
ammonium by GLN2 would occur largely due to bypassing nitrate reduction as the rate-limiting step for $\mathrm{N}$ assimilation. The resultant increase in the ratio of Gln to Glu content (Fig. 4c) corresponds to the preferential enhancement of the protonproducing GLN reaction over the proton-consuming GOGAT reaction. The metabolic imbalances exerted by the GLN2 reaction and GLN2-related pathways could lead to the production of large amounts of protons in shoot cells that stimulate proton effluxes to the apoplasm; however, the volume of the shoot apoplasm is probably too small to accommodate such a large proton efflux. Thus, in the presence of toxic levels of ammonium, the GLN2 reaction causes acidic stress inside and outside the cells and triggers acidic stress responses that modulate gene expression (Fig. 5 and Supplementary Fig. 11). Given that the wild-type when grown at a higher $\mathrm{pH}$ phenocopies the ammoniuminsensitive lines at lower $\mathrm{pH}$, the acidic stress-sensitive mutants show ammonium-hypersensitivity, and proton-consuming nitrate reduction alleviates ammonium toxicity (Fig. 6), we conclude that acidic stress is one of the primary causes for ammonium toxicity in A. thaliana. In this framework, upregulation of Arabidopsis NIA1 and NIA2 genes encoding nitrate reductase (NR) 25,26 and activation of spinach $\mathrm{NR}^{29}$ responding to acidic stress are understandable regulatory responses in the context of maintaining cellular $\mathrm{pH}$ homeostasis.

The present study does not address how ammoniumdependent acidification triggers growth deficiency at the cellular and subcellular scales. The chloroplastic localization of GLN2 indicates that proton production must occur within chloroplasts in the elevated ammonium condition. A previous study reported abnormal chloroplast membrane structure including swollen compartments at late stages of ammonium toxicity ${ }^{30}$; however, we did not find any similar structural changes in the shoots of ammonium-grown plants (Supplementary Fig. 13a), where the intermediates of the Calvin-Benson cycle were not depleted compared with nitrate-grown shoots (Supplementary Fig. 13b). These observations do not support a deficiency in chloroplast function as a primary cause of ammonium toxicity. Apoplastic $\mathrm{pH}$ in sunflower leaves and cytosolic $\mathrm{pH}$ in carrot cell suspensions decrease after application of millimolar levels of ammonium ${ }^{31,32}$. The ammonium-inducible genes whose expression is downregulated by GLN2 deficiency, PGIP1 and PGIP2 (Supplementary Fig. 11a), contribute to cell wall stabilization under acidic stress ${ }^{27}$, implying apoplastic acidification as a target of ammonium toxicity. On the other hand, in the presence of toxic levels of ammonium, the expression of GABA shunt-related genes (Supplementary Fig. 11a), the molar ratio of GABA to Glu (Supplementary Fig. 11b), and oxygen uptake rates ${ }^{33}$ are increased as biochemical $\mathrm{pH}$-stats ${ }^{26,34}$ that may represent an intracellular acidic burden. Given that changes in $\mathrm{pH}$ environments influence a wide spectrum of physiological processes, elucidating the relationship between ammonium-dependent acidification and growth deficiency awaits future study.

At the whole-plant scale, our grafting work demonstrated that root GLN1;2 activity attenuates ammonium toxicity in the shoots, whilst shoot GLN2 activity causes the condition (Fig. 3). Considering that GLN1;2 is the ammonium-inducible low-affinity enzyme expressed in the epidermis and cortex of roots, and its deficiency elevates ammonium levels in xylem sap when ammonium is supplied ${ }^{20}$, root GLN1;2 could act as a barrier to prevent the shoot-to-root transport of ammonium, thus avoiding ammonium assimilation by shoot GLN2. In oilseed rape plants, replacing $3 \mathrm{mM}$ nitrate in a nutrient solution with $10 \mathrm{mM}$ ammonium increased the ammonium levels in xylem sap linearly with time, attaining concentrations $>5 \mathrm{mM}^{35}$, which could indicate breaking through the barrier. On the other hand, we observed that shoot expression of GLN1;2 and GLN1;3 encoding low-affinity GLN1 isozymes was significantly larger under $10 \mathrm{mM}$ ammonium than $10 \mathrm{mM}$ nitrate (Supplementary Fig. 3b). Moreover, a larger protein signal corresponding to shoot GLN1s was detected when plants received ammonium rather than nitrate nutrition (Fig. 2c and Supplementary Fig. 14a, b), implying the above-mentioned barrier function of GLN1;2 and GLN1;3 in the shoot. Whilst a large activity of shoot GLN1s could reduce ammonium assimilation by shoot GLN2, shoot GLN1s reaction per se may produce an excessive proton, thereby causing ammonium toxicity. Thus, we compared the proton concentrations of water extracts from the Col, ami2, and $g \ln 1 ; 2 \operatorname{gln} 1 ; 3$ shoots incubated in media containing $10 \mathrm{mM}$ ammonium (Supplementary Fig. 14c). Interestingly, we found that double knockout of GLN1;2 and GLN1;3 did not significantly reduce the proton concentrations, whereas it decreased shoot GS activity by ca. 35\%, being comparable to GLN2 deficiency (Supplementary Fig. 14c, d). This suggests that shoot GLN1;2 and GLN1;3 could assimilate excessive ammonium without causing proton accumulation in the shoot, supporting their barrier function. It is a challenging issue to address how GLN1s and GLN2 have different effects on $\mathrm{pH}$ regulation in the future.

It is widely believed that the plastidic isoform of GS (GS2) plays a central role in re-assimilating photorespiratory ammonium. The mutants lacking GS2 in barley cannot survive under photorespiratory air conditions ${ }^{36}$. In Lotus japonicus, the leaves of GS2-deficient mutants show severe necrotic phenotype when transferred from high to low $\mathrm{CO}_{2}$ condition ${ }^{37}$. On the other hand, a recent study reported that Arabidopsis GS2-deficient mutants can complete their life cycle under air conditions, albeit smaller and slightly chlorotic than the wild-type ${ }^{17}$. Even under conditions that lower photorespiration, the GLN2-deficient mutants show growth impairment, suggesting an important role of GLN2 in primary nitrogen assimilation. Also, we did not find a strong suppression of vegetative growth of ami2 and $g \ln 2$ plants grown in the soil under photorespiratory conditions compared with the wild-type (Supplementary Fig. 15a), although their seedling growth during 1 week after imbibition in the halfstrength Murashige and Skoog media was significantly retarded (Supplementary Fig. 15b, c). Moreover, ami2 and gln 2 plants propagated the seeds whose total $\mathrm{N}$ and $\mathrm{C}$ concentrations and germination rates were comparable to those of the wild-type (Supplementary Fig. 15d-f). Thus, in A. thaliana, GLN2, GLN1s, and GDHs proteins could redundantly contribute to the reassimilation of photorespiratory ammonia. This may be why no mutant lacking GLN2 has been isolated as a photorespiratory mutant from Arabidopsis plants. On the other hand, the present study did not clarify how improved ammonium insensitivity in Arabidopsis GLN2-deficient mutants is related to photorespiration. To elucidate this, it awaits many efforts in comparison between photorespiratory and non-photorespiratory conditions.

The present study demonstrated that GLN2 and NRT1.1 reduce ammonium tolerance via separate mechanisms when plants experience high ammonium conditions (Supplementary Fig. 12). By contrast, these genes are nitrate-inducible genes that are crucial for plant adaptation to nitrate-dominant environments ${ }^{38,39}$. This observation suggests that the adaptive traits to nitrate and ammonium could be exclusive, and therefore, breeding ammonium-tolerant crops might sacrifice their adaptability to nitrate. Arabidopsis growth is enhanced by elevating $\mathrm{CO}_{2}$ more remarkably with ammonium as the sole $\mathrm{N}$ source than with nitrate, although, even under elevated $\mathrm{CO}_{2}$, the absolute value of plant biomass is still greater when receiving nitrate ${ }^{1}$. Overcoming ammonium toxicity could lead to elevated $\mathrm{CO}_{2}$-adapted crops in terms of their mode of $\mathrm{N}$ utilization. 


\section{Methods}

Plant materials and growth conditions. A. thaliana, accession Columbia (Col) was the control line used in this study. The T-DNA insertion mutants $g \ln 2$ $\left(\right.$ SALK_051953), $g \ln 2-2\left(\right.$ SALK_071292) ${ }^{17}, g \ln 1 ; 2-1$ (SALK_145235) ${ }^{19}, g \ln 1 ; 2-2$ $\left(\text { SALK_102291) }{ }^{18} \text {, stop1-KO (SALK_114108) }\right)^{26}$, and $n r t 1.1$ (SALK_097431) ${ }^{28}$ were purchased from the European Arabidopsis Stock Centre. The Arabidopsis FOX lines ${ }^{12}$ and the ethyl methanesulfonate-mutagenized stop1 mutant (psi00011) ${ }^{26}$ were obtained from the RIKEN BioResource Research Center. Seeds of the NR-null mutant ${ }^{14}$ and $g \ln 1 ; 2 \operatorname{gln} 1 ; 3$ mutant ${ }^{20}$ were acquired from Dr. Nigel M. Crawford (University of California, San Diego) and Dr. Soichi Kojima (Tohoku University, Sendai), respectively.

Seeds were surface-sterilized and sown in plastic Petri dishes (diameter $90 \mathrm{~mm}$, depth $20 \mathrm{~mm}$, Iwaki, Tokyo, Japan) containing about 30-mL N-modified Murashige and Skoog medium, supplemented with $10 \mathrm{mM} \mathrm{MES,} 2 \%(\mathrm{w} / \mathrm{v})$ sucrose, and $0.25 \%(\mathrm{w} / \mathrm{v})$ gellan gum ( $\mathrm{pH} 5.7)$. The $\mathrm{N}$ sources were $10 \mathrm{mM} \mathrm{KNO}_{3}(10 \mathrm{mM}$ nitrate condition), $5 \mathrm{mM} \mathrm{NH}_{4} \mathrm{NO}_{3}$ with $10 \mathrm{mM} \mathrm{KCl}(5 \mathrm{mM}$ ammonium nitrate condition), or $5 \mathrm{mM}\left(\mathrm{NH}_{4}\right)_{2} \mathrm{SO}_{4}$ with $10 \mathrm{mM} \mathrm{KCl}(10 \mathrm{mM}$ ammonium condition). The seeds were placed in the dark at $4{ }^{\circ} \mathrm{C}$ for $3 \mathrm{~d}$. Plants were grown in a horizontal position under a photosynthetic photon flux density of $100-130 \mu \mathrm{mol} \mathrm{m}^{-2} \mathrm{~s}^{-1}(16$ $\mathrm{h}$ light $/ 8 \mathrm{~h}$ dark cycle) at $23^{\circ} \mathrm{C}$. For the transfer experiments, surface-sterilized seeds were sown in plastic Petri dishes (length, $140 \mathrm{~mm}$; width, $100 \mathrm{~mm}$; depth, 20 $\mathrm{mm}$; Eiken Chemical Co. Ltd., Taito-ku, Tokyo, Japan) containing 50-mL halfstrength $\mathrm{N}$-modified Murashige and Skoog medium containing $2.5 \mathrm{mM}$ ammonium as the sole $\mathrm{N}$ source, supplemented with $4.7 \mathrm{mM}$ MES, $1 \%$ (w/v) sucrose, and $0.4 \%(\mathrm{w} / \mathrm{v})$ gellan gum $(\mathrm{pH} 6.7)^{40}$. This culture condition allowed almost uniform growth of different lines, alleviating ammonium toxicity. The seeds were placed in the dark at $4{ }^{\circ} \mathrm{C}$ for $3 \mathrm{~d}$. The plants were grown in a vertical position for $7 \mathrm{~d}$ under a photosynthetic photon flux density of $100-130 \mu \mathrm{mol} \mathrm{m}^{-2} \mathrm{~s}^{-1}(16 \mathrm{~h}$ light $/ 8 \mathrm{~h}$ dark cycle) at $23^{\circ} \mathrm{C}$. The plants were transferred with sterilized tweezers to varying $\mathrm{N}$ conditions and grown in a horizontal position for further experiments. Other details of plantlet cultivation are presented in the Results section and the figure legends.

Isolation of ammonium-insensitive lines. Forty-five seedlings per plate of Arabidopsis FOX lines ${ }^{12}$ were grown on the above-mentioned N-modified Murashige and Skoog media containing $5 \mathrm{mM}\left(\mathrm{NH}_{4}\right)_{2} \mathrm{SO}_{4}$ with $10 \mathrm{mM} \mathrm{KCl}$ as the sole $\mathrm{N}$ source for $11 \mathrm{~d}$ in a horizontal position. The ammonium-insensitive candidate lines were visually selected based on shoot size and color of the cotyledons or true leaves. Then, the next generation of each candidate line was obtained to refresh the seeds. In the second screen, the ammonium insensitivity of the $10 \mathrm{mM}$ ammoniumgrown 11-d-old line was evaluated quantitatively by determining their seedling fresh weights in comparison with the wild-types (more than 10 biological replicates). The lines having statistically significant increases in the fresh weight compared with the wild-types were regarded as the ammonium-insensitive ones. In total, seed mixtures that included 15,800 lines (pss10001-10016) were used for a series of screens, resulting in the isolation of three lines (ami1-ami3). The transgene included in the candidate line was recovered in a vector (pCR8/GW/TOPO, ThermoFisher Scientific, Waltham, MA, USA) using specific primers ${ }^{12}$ and identified by sequencing. The expression of the native and transgene was checked by RT-PCR and qRT-PCR. The identified genes for amil and ami3 were different from those for ami2.

Extraction of total RNA. Shoots and roots were harvested, immediately frozen with liquid $\mathrm{N}_{2}$, and stored at $-80^{\circ} \mathrm{C}$ until use. Frozen samples were ground with a Multi-Beads Shocker (Yasui Kikai Corp., Osaka Prefecture, Osaka, Japan) using zirconia beads (diameter, $5 \mathrm{~mm}$ ). Total RNA was extracted using the RNeasy Plant Mini Kit (Qiagen) and on-column DNase digestion according to the manufacturer's instructions.

Reverse transcription and quantitative (real-time) PCR. Reverse transcription was performed using a ReverTra Ace qPCR RT Master Mix with gDNA Remover (Toyobo Co. Ltd., Tokyo, Japan) according to the manufacturer's instructions. The synthesized cDNA was diluted 10 -fold with distilled water and used in quantitative PCR (qPCR). Transcript levels were measured using a StepOnePlus Real-Time PCR System (ThermoFisher Scientific). The obtained cDNA $(2 \mu \mathrm{L})$ was amplified in the presence of 10- $\mu \mathrm{L}$ KAPA SYBR FAST qPCR Kit (Nippon Genetics Co. Ltd., Tokyo, Japan), $0.4-\mu \mathrm{L}$ specific primers $(0.2 \mu \mathrm{M}$ final concentration), and $7.2-\mu \mathrm{L}$ sterile water. Transcript levels were quantified using a relative standard curve with ACTIN3 as the internal standard. A dilution series of total cDNAs were used as templates to generate the standard curves. The primer sequences used in the experiments are shown in Supplementary Data 3.

Immunoblot analysis of GLN isoproteins. Immunoblot analysis of GLN proteins was performed based on a previously described method ${ }^{40}$. Shoots were harvested, frozen with liquid $\mathrm{N}_{2}$, and stored at $-80^{\circ} \mathrm{C}$ until use. Frozen samples were ground with a Multi-Beads Shocker (Yasui Kikai Corp.) using zirconia beads (diameter, 5 $\mathrm{mm})$. Total proteins were extracted with 10 volumes of sample buffer $[2 \%(\mathrm{w} / \mathrm{v})$ SDS, $62.5 \mathrm{mM}$ Tris- $\mathrm{HCl}(\mathrm{pH} 6.8), 7.5 \%$ (v/v) glycerol, $50 \mathrm{mM}$ DTT and $0.01 \%(\mathrm{w} /$ v) bromophenol blue] containing a protease inhibitor tablet (Roche Diagnostics,
Basel, Switzerland). The extracts were incubated at $95^{\circ} \mathrm{C}$ for 5 min followed by cooling on ice and centrifugation at $20,400 \times g$ at room temperature $\left(20-25^{\circ} \mathrm{C}\right)$ for $10 \mathrm{~min}$. A $10-\mu \mathrm{L}$ aliquot of the supernatant (equivalent to approximately $1-\mathrm{mg}$ fresh sample) was subjected to SDS-PAGE in a $12 \%(\mathrm{w} / \mathrm{v})$ gel (TGX FastCast Acrylamide Kit, Bio-Rad Laboratories, Hercules, CA, USA) and transferred to a PVDF membrane (Trans-Blot Turbo Mini PVDF Transfer Packs, Bio-Rad Laboratories, Hercules, CA, USA) using HIGH MW with a semi-dry blotting system (Trans-Blot Turbo Transfer System, Bio-Rad Laboratories). The membrane was incubated overnight in blocking buffer containing 5\% (w/v) ECL Prime Blocking Agent (GE Healthcare, Little Chalfont, UK), 0.1\% (v/v) Tween-20, $50 \mathrm{mM}$ Tris- $\mathrm{HCl}$, and $150 \mathrm{mM} \mathrm{NaCl}$ ( $\mathrm{pH}$ 7.6). The blocked membrane was then incubated for $1 \mathrm{~h}$ with a 1/10,000 dilution of the polyclonal antibodies raised against maize cytosolic glutamine synthetase ${ }^{15}$ that was obtained from Dr. Hitoshi Sakakibara (Nagoya University, Nagoya, Aichi, Japan). After rinsing with a buffer containing $0.1 \%$ Tween-20, $50 \mathrm{mM}$ Tris- $\mathrm{HCl}$, and $150 \mathrm{mM} \mathrm{NaCl}(\mathrm{pH}$ 7.6), the antigen-antibody complex was detected using a 1/100,000 dilution of horseradish peroxidase-conjugated to goat anti-rabbit IgG (NA935, GE Healthcare) and visualized by chemiluminescent detection (ECL Prime, GE Healthcare) using ImageQuant LAS 3000 mini (Fujifilm, Tokyo, Japan). The signal intensities for each band corresponding to GLN1s and GLN2 isoproteins were quantified using Image J software, version 10.2. After detection, the membrane was stained with the GelCode Blue Stain Reagent (ThermoFisher Scientific).

Microarray analysis. Total RNA was extracted as described above. RNA quality was assessed using an Agilent 2100 bioanalyzer (Agilent Technologies). RNA amplification, labeling, hybridization, and scanning with the $3^{\prime}$ IVT Express Kit (Affymetrix) and the GeneChip Arabidopsis Genome ATH1 Array (Affymetrix) were conducted according to the manufacturer's instructions. The data set from the microarray chips was normalized by the Microarray Suite 5.0 (MAS5) method (Affymetrix). When the signal detection of a transcript was labeled "Absent" or "Marginal", the sequence was omitted from the subsequent quantitative analysis. The raw data are shown in Supplementary Data 4. Changes in respective gene expression levels between $\mathrm{Col}$ and ami2 were represented as logarithms to base 2 of the ratios in signal intensities.

Grafting between shoots and roots. Arabidopsis seedlings were grown for $4 \mathrm{~d}$ on media containing $2.5 \mathrm{mM}$ ammonium as the sole $\mathrm{N}$ source at $\mathrm{pH} 6.7^{40}$ before grafting. Each seedling was perpendicularly cut at the hypocotyl with the tip of an injection needle (NN-2613S, TERUMO, Tokyo, Japan) on a mixed cellulose membrane (HAWP09000, MERCK MILLIPORE, Darmstadt, Germany). The scion was kept in touch with the rootstock through a section of $0.4 \mathrm{~mm}$ diameter silicon tubing as described ${ }^{41}$. The grafted plants were grown for another $4 \mathrm{~d}$ at $27^{\circ} \mathrm{C}$, and then, for $2 \mathrm{~d}$ at $23^{\circ} \mathrm{C}$. Plants without adventitious roots were transferred to the fresh media and further grown and used for analyses of growth and gene expression.

Determination of glutamine synthetase activity. GLN activity was determined as the ADP-dependent conversion rate of L-glutamine to $\gamma$-glutamylhydroxamate ${ }^{42}$. Shoots and roots were harvested, immediately frozen with liquid $\mathrm{N}_{2}$, and stored at $-80^{\circ} \mathrm{C}$ until use. Frozen samples were ground with a TissueLyser II (QIAGEN) using $5 \mathrm{~mm}$ zirconia beads. The powder was mixed with 10 volumes of extraction buffer (100 mM Tris-HCl, pH 7.5, 1\% (w/v) PVP-40, 1 mM EDTA, 1 mM MnCl , $0.5 \%(\mathrm{v} / \mathrm{v}) \beta$-mercaptoethanol, $0.1 \mathrm{mM} 4$-APMSF). The extracts were centrifuged at $12,000 \times g$ at $4{ }^{\circ} \mathrm{C}$ for $10 \mathrm{~min}$. The reaction was started by adding $45 \mu \mathrm{l}$ of preincubated assay buffer $(40 \mathrm{mM}$ imidazole- $\mathrm{HCl}, \mathrm{pH} 7.0,20 \mathrm{mM}$ sodium arsenate, 0.5 $\mathrm{mM}$ ADP, $3 \mathrm{mM} \mathrm{MnCl}, 60 \mathrm{mM} \mathrm{NH}_{2} \mathrm{OH}, 30 \mathrm{mM} \mathrm{L}$-glutamine) to $5 \mu \mathrm{L}$ of the supernatant. The mixture was incubated at $30^{\circ} \mathrm{C}$ for $15 \mathrm{~min}$. The reaction was stopped by adding $30 \mu \mathrm{l}$ of $\mathrm{FeCl}_{3}-\mathrm{TCA}-\mathrm{HCl}$ solution $\left(2.6 \% \mathrm{FeCl}_{3} \cdot 6 \mathrm{H}_{2} \mathrm{O}, 4 \%\right.$ trichloroacetic acid in $1 \mathrm{~N} \mathrm{HCl}$ ). Ferric $\gamma$-glutamylhydroxamic acid was measured by spectrophotometric absorbance at $540 \mathrm{~nm}$.

Determination of amino acids and photosynthetic intermediates. Amino acids and photosynthetic intermediates were extracted and their concentrations were determined based on the method reported by Miyagi et al. $(2010)^{43}$ with minor modifications. Shoots were harvested, frozen with liquid $\mathrm{N}_{2}$, and stored at $-80^{\circ} \mathrm{C}$ until use. Frozen samples were ground with a Multi-Beads Shocker (Yasui Kikai Corp.) using zirconia beads (diameter, $5 \mathrm{~mm}$ ). Metabolites were extracted with $50 \%$ (v/v) methanol containing $50 \mu \mathrm{M}$ PIPES and $50 \mu \mathrm{M}$ methionine sulfone (internal standard). After the first centrifugation $\left(21,500 \times g, 5 \mathrm{~min}, 4^{\circ} \mathrm{C}\right)$, the supernatant was transferred to a $3 \mathrm{kDa}$ cut-off filter (UFC500324, Millipore, Billerica, MA, USA) and centrifuged again $\left(13,700 \times g, 30 \mathrm{~min}, 4^{\circ} \mathrm{C}\right)$. The filtered extract was used for capillary electrophoresis-mass spectrometry (CE-MS) analysis.

Measurements of metabolites were performed using an Agilent 6400 series triple quadrupole CE-MS system (CE; 7100 Capillary Electrophoresis, MS; 6420 Triple Quad LC/MS, Agilent Technologies, Santa Clara, CA, USA) in multi reaction monitoring (MRM) mode ${ }^{43,44}$. A DB-WAX capillary (polyethylene glycolcoated, $100 \mathrm{~cm} \times 50 \mu \mathrm{m}$ i.d., Agilent Technology) with $20 \mathrm{mM}$ ammonium acetate $(\mathrm{pH} 8.5)$ as the running buffer was used for measuring anionic compounds (e.g., organic acids and phosphorylated compounds), and an uncoated fused silica 
capillary (100 cm $\times 50 \mu \mathrm{m}$ i.d., GL Sciences, Tokyo, Japan) with $1 \mathrm{M}$ formic acetate was used for cationic compounds (e.g., amino acids). MS analysis at the applied $-25 \mathrm{kV}$ for anions was carried out in negative ion mode. Cations were determined in positive ion mode (applied voltage $25 \mathrm{kV}$ ). For MS stabilization, $5 \mathrm{mM}$ ammonium acetate (for anions) or $0.1 \%$ formic acid (v/v) (for cations) in $50 \%(\mathrm{v} / \mathrm{v}$ ) methanol was used as sheath solution, applied to the capillary at $10 \mu \mathrm{L} \mathrm{min}{ }^{-1}$ using an isocratic HPLC pump (Agilent 1200 series) equipped with a 1:100 splitter. The capillary voltage $( \pm 3500 \mathrm{~V})$ and the drying nitrogen gas $\left(\right.$ at $\left.320^{\circ} \mathrm{C}\right)$ flow $(8 \mathrm{~L}$ $\mathrm{min}^{-1}$ ) were held constant for approximately $30 \mathrm{~min}$ during each electrophoresis run. Quantitative accuracy was determined using known concentrations of standard reference compounds using Agilent MassHunter Software, version B.07.00.

Determination of ammonium. Ammonium was extracted and its concentration was determined with slight modifications to the method reported by Hachiya et al. ${ }^{42}$. Shoots were harvested, immediately frozen with liquid $\mathrm{N}_{2}$, and stored at $-80^{\circ} \mathrm{C}$ until use. Frozen samples were ground with a Multi-Beads Shocker (Yasui Kikai Corp.) using zirconia beads (diameter, $5 \mathrm{~mm}$ ). One $\mathrm{mL}$ of $0.1 \mathrm{~N} \mathrm{HCl}$ and 500 $\mu \mathrm{L}$ of chloroform were added to the frozen powder, followed by vortexing for 15 min. The mixture was centrifuged at $12,000 \times g$ at $8^{\circ} \mathrm{C}$ for $10 \mathrm{~min}$. The aqueous phase was transferred to a microtube containing $50 \mathrm{mg}$ of acid-washed activated charcoal (No. 035-18081; Wako, Osaka, Japan). The mixture was vortexed and centrifuged at $20,400 \times g$ at $8^{\circ} \mathrm{C}$ for $10 \mathrm{~min}$. The ammonium content of the supernatant was spectroscopically determined using an Ammonia Test Kit (No. 277-14401, Wako) according to the manufacturer's instructions.

Determination of $\mathbf{H}^{+}$concentration in a water extract of shoots. For Fig. $5 \mathrm{c}$ and Supplementary Fig. 14c, plants grown on media containing $10 \mathrm{mM}$ ammonium or $10 \mathrm{mM}$ nitrate were submerged in $5 \mathrm{~mL}$ of the corresponding liquid medium without MES buffer in the presence or absence of $1 \mathrm{mM}$ methionine sulfoximine (MSX). The plants were returned to their original positions on each medium and incubated for $5 \mathrm{~h}$ in the light. Shoots were harvested, immediately frozen with liquid $\mathrm{N}_{2}$, and stored at $-80^{\circ} \mathrm{C}$ until use. For Fig. 6a and Supplementary Fig. 11f, the shoots grown on media containing $10 \mathrm{mM}$ ammonium or 10 $\mathrm{mM}$ nitrate were harvested without prior incubation in liquid media, immediately frozen with liquid $\mathrm{N}_{2}$, and stored at $-80{ }^{\circ} \mathrm{C}$ until use. Ten volumes of $\mathrm{H}_{2} \mathrm{O}$ for Fig. $5 \mathrm{c}$ and Supplementary Figs. 11f, $14 \mathrm{c}$ and 40 volumes of $\mathrm{H}_{2} \mathrm{O}$ for Fig. 6 a were added to the frozen powder followed by centrifugation at $10,000 \times g$ at room temperature for $10 \mathrm{~min}$. The $\mathrm{pH}$ of the supernatant was measured with a portable $\mathrm{pH}$ meter (B212, Horiba, Ltd.). The values were converted to proton concentrations.

Determination of $\mathbf{H}^{+}$efflux from shoots. Excised shoots from the plants grown on media containing $10 \mathrm{mM}$ ammonium or $10 \mathrm{mM}$ nitrate were submerged in 100 volumes of the corresponding liquid medium without MES buffer in the presence or absence of $1 \mathrm{mM}$ methionine sulfoximine (MSX). The submerged shoots were incubated in liquid media for $5 \mathrm{~h}$ in the light. The $\mathrm{pH}$ changes in the incubation media were measured with a portable $\mathrm{pH}$ meter (B212, Horiba, Ltd.). The changes in $\mathrm{pH}$ were converted to changes in proton concentrations.

Determination of $\mathbf{H}^{+}$efflux from mesophyll protoplasts. Mesophyll protoplasts were prepared with slight modifications to the method reported by Endo et al. ${ }^{45}$. In one dish, 20 plants were grown for $7 \mathrm{~d}$ on media containing $2.5 \mathrm{mM}$ ammonium as the sole $\mathrm{N}$ source at $\mathrm{pH} 6.7^{40}$ in a vertical position. Ten plants were transferred to fresh medium with the same composition and grown for another $7 \mathrm{~d}$. Two plants were further transferred to a medium containing $10 \mathrm{mM}$ ammonium and grown for $3 \mathrm{~d}$ in a horizontal position. The adaxial epidermal surface was affixed to plastic tape, whereas the abaxial epidermal surface was attached to a strip of 15 -mm wide Scotch transparent tape ( $3 \mathrm{M}$, St. Paul, MN, USA). The Scotch tape was then carefully pulled away from the plastic tape, peeling away the abaxial epidermal surface cell layer. The plastic tape samples with adhering peeled leaves were transferred to $1.5 \mathrm{~mL}$ microtubes containing $1 \mathrm{~mL}$ of an enzyme solution $[0.75 \%$ (w/v) cellulase "Onozuka" R10 (Yakult, Tokyo, Japan), 0.25\% (w/v) macerozyme “Onozuka” R10 (Yakult), 0.4 M mannitol, $8 \mathrm{mM} \mathrm{CaCl}_{2}$, and $5 \mathrm{mM} \mathrm{MES,} \mathrm{pH} \mathrm{5.6].}$ The tube was slowly rotated for $20 \mathrm{~min}$ at room temperature. The liberated protoplasts were filtered through a $30-\mu \mathrm{m}$ nylon filter (NY30-HD, SEMITEC, Osaka, Japan), and collected by centrifugation at $100 \times g$ at $4{ }^{\circ} \mathrm{C}$ for $5 \mathrm{~min}$. The protoplast suspensions of leaf samples from 16 plants were pooled. The supernatant was removed, and the protoplasts were resuspended in $1 \mathrm{~mL}$ of a wash solution $[0.4 \mathrm{M}$ mannitol and $8 \mathrm{mM} \mathrm{CaCl}_{2}, \mathrm{pH} 6.5$ ] followed by centrifugation at $100 \times g$ at $4{ }^{\circ} \mathrm{C}$ for $5 \mathrm{~min}$. This washing step was repeated once more, after which the protoplasts were suspended in $100 \mu \mathrm{L}$ of the wash solution. Ten $\mu \mathrm{L}$ of protoplast suspension was mixed with $90 \mu \mathrm{L}$ of an assay solution $\left[0.4 \mathrm{M}\right.$ mannitol, $8 \mathrm{mM} \mathrm{CaCl}_{2}, 0.02 \%$ bromocresol purple (BCP) $\mathrm{pH}$ 6.5] supplemented with $5 \mathrm{mM}\left(\mathrm{NH}_{4}\right)_{2} \mathrm{SO}_{4}$ and $10 \mathrm{mM}$ $\mathrm{KCl}$ in the presence or absence of $1 \mathrm{mM} \mathrm{MSX}, 10 \mathrm{mM} \mathrm{KCl}$, or $10 \mathrm{mM} \mathrm{KNO}_{3}$. The mixture was incubated at $23^{\circ} \mathrm{C}$ in the light until color changes were observed.

Observation of chloroplast ultrastructure in mesophyll cells with TEM. For ultrastructural analyses, true leaves from $\mathrm{Col}, a m i 2$, and $g \ln 25 \mathrm{~d}$ after the transfer to media containing $10 \mathrm{mM}$ ammonium or $10 \mathrm{mM}$ nitrate were analyzed by transmission electron microscopy according to a previously described method ${ }^{46}$ with modification. Leaves were fixed with $4 \%(\mathrm{w} / \mathrm{v})$ paraformaldehyde and $2 \%(\mathrm{v} / \mathrm{v})$ glutaraldehyde in $50 \mathrm{mM}$ sodium cacodylate buffer ( $\mathrm{pH} 7.4$ ) overnight at $4{ }^{\circ} \mathrm{C}$. After the samples were post-fixed with $1 \%(\mathrm{w} / \mathrm{v})$ osmium tetroxide for $3 \mathrm{~h}$ and dehydrated in a graded methanol series, the samples were embedded in EPON812 resin. Micrographs were recorded using a JEM-1400 (JEOL) transmission electron microscope.

Statistical and cluster analyses. All statistical analyses were conducted using $\mathrm{R}$ software, version 3.6.2. Two-sided tests were used for Welch's $t$-test. Adjustments were made for Tukey-Kramer's multiple comparison test. Hierarchical clustering was performed using Gene Cluster software, version 3.0, with "Correlation (uncentered)" as the similarity metric and "Average linkage" as the clustering method. The results were visualized using Java TreeView software, version 1.1.6r4 Other details of analyses are provided in the "Results" section and in the table and figure legends.

\section{Data availability}

The raw microarray data used in this study are available in the ArrayExpress database at EMBL-EBI under accession number E-MTAB-10796. Source data are provided with this paper.

Received: 19 September 2019; Accepted: 29 July 2021; Published online: 16 August 2021

\section{References}

1. Rubio-Asensio, J. S. \& Bloom, A. J. Inorganic nitrogen form: a major player in wheat and Arabidopsis responses to elevated $\mathrm{CO}_{2}$. J. Exp. Bot. 68, 2611-2625 (2016).

2. Kant, S., Bi, Y. M. \& Rothstein, S. J. Understanding plant response to nitrogen limitation for the improvement of crop nitrogen use efficiency. J. Exp. Bot. 62, 1499-1509 (2011)

3. Britto, D. T. \& Kronzucker, H. J. $\mathrm{NH}_{4}{ }^{+}$toxicity in higher plants: a critical review. J. Plant Physiol. 159, 567-584 (2002).

4. Li, B., Li, G., Kronzucker, H. J., Baluška, F. \& Shi, W. Ammonium stress in Arabidopsis signaling, genetic loci, and physiological targets. Trends Plant Sci. 19, 107-114 (2014)

5. Esteban, R., Ariz, I., Cruz, C. \& Moran, J. F. Mechanisms of ammonium toxicity and the quest for tolerance. Plant Sci. 248, 92-101 (2016).

6. Hachiya, T. et al. Nitrate addition alleviates ammonium toxicity without lessening ammonium accumulation, organic acid depletion and inorganic cation depletion in Arabidopsis thaliana shoots. Plant Cell Physiol. 53, 577-591 (2012).

7. Qin, C. et al. GDP-mannose pyrophosphorylase is a genetic determinant of ammonium sensitivity in Arabidopsis thaliana. Proc. Natl Acad. Sci. USA 105, 18308-18313 (2008)

8. Tanaka, H. et al. Identification and characterization of Arabidopsis AtNUDX9 as a GDP-d-mannose pyrophosphohydrolase: its involvement in root growth inhibition in response to ammonium. J. Exp. Bot. 66, 5797-5808 (2015).

9. Li, B. et al. Arabidopsis plastid metalloprotease AMOS1/EGY1 integrates with ABA signaling to regulate global gene expression in response to ammonium stress. Plant Physiol. 160, 2040-2051 (2012).

10. $\mathrm{Li}, \mathrm{G}$. et al. The Arabidopsis AMOT1/EIN3 gene plays a important role in the amelioration of ammonium toxicity. J. Exp. Bot. 70, 1375-1388 (2019).

11. Jian, S. et al. NRT1.1-related $\mathrm{NH}_{4}{ }^{+}$toxicity is associated with a disturbed balance between $\mathrm{NH}_{4}{ }^{+}$uptake and assimilation. Plant Physiol. 178, 1473-1488 (2018)

12. Ichikawa, T. et al. The FOX hunting system: an alternative gain-of-function gene hunting technique. Plant J. 48, 974-985 (2006).

13. Patterson, $K$. et al. Distinct signalling pathways and transcriptome response signatures differentiate ammonium- and nitrate-supplied plants. Plant Cell Environ. 33, 1486-1501 (2010).

14. Wang, R., Xing, X. \& Crawford, N. Nitrite acts as a transcriptome signal at micromolar concentrations in Arabidopsis roots. Plant Physiol. 145, 1735-1745 (2007)

15. Sakakibara, H., Kawabata, S., Hase, T. \& Sugiyama, T. Differential effects of nitrate and light on the expression of glutamine synthetases and ferredoxindependent glutamate synthase in maize. Plant Cell Physiol. 33, 1193-1198 (1992).

16. Cheng, X. F. \& Wang, Z. Y. Overexpression of COL9, a CONSTANS-LIKE gene, delays flowering by reducing expression of $C O$ and FT in Arabidopsis thaliana. Plant J. 43, 758-768 (2005). 
17. Ferreira, S., Moreira, E., Amorim, I., Santos, C. \& Melo, P. Arabidopsis thaliana mutants devoid of chloroplast glutamine synthetase (GS2) have nonlethal phenotype under photorespiratory conditions. Plant Physiol. Biochem. 144, 365-374 (2019).

18. Lothier, J. et al. The cytosolic glutamine synthetase GLN1;2 plays a role in the control of plant growth and ammonium homeostasis in Arabidopsis rosettes when nitrate supply is not limiting. J. Exp. Bot. 62, 1375-1390 (2011).

19. Guan, M., Møller, I. S. \& Schjoerring, J. K. Two cytosolic glutamine synthetase isoforms play specific roles for seed germination and seed yield structure in Arabidopsis. J. Exp. Bot. 66, 203-212 (2015).

20. Konishi, N. et al. Contributions of two cytosolic glutamine synthetase isozymes to ammonium assimilation in Arabidopsis roots. J. Exp. Bot. 68, 613-625 (2017).

21. Thieme, C. J. et al. Endogenous Arabidopsis messenger RNAs transported to distant tissues. Nat. Plants 1, 15025 (2015).

22. Bittsánszky, A., Pilinszky, K., Gyulai, G. \& Komives, T. Overcoming ammonium toxicity. Plant Sci. 231, 184-190 (2015).

23. Sato, S. \& Yanagisawa, S. Characterization of metabolic states of Arabidopsis thaliana under diverse carbon and nitrogen nutrient conditions via targeted metabolomic analysis. Plant Cell Physiol. 55, 306-319 (2014).

24. Britto, D. T. \& Kronzucker, H. J. Nitrogen acquisition, PEP carboxylase, and cellular pH homeostasis: new views on old paradigms. Plant Cell Environ. 28, 1396-1409 (2005).

25. Lager, I. et al. Changes in external $\mathrm{pH}$ rapidly alter plant gene expression and modulate auxin and elicitor responses. Plant Cell Environ. 33, 1513-1528 (2010).

26. Sawaki, Y. et al. STOP1 regulates multiple genes that protect Arabidopsis from proton and aluminum toxicities. Plant Physiol. 150, 281-294 (2009).

27. Kobayashi, Y. et al. STOP2 activates transcription of several genes for Al- and Low $\mathrm{pH}$-tolerance that are regulated by STOP1 in Arabidopsis. Mol. Plant 7, 311-322 (2014).

28. Hachiya, T. et al. Evidence for a nitrate-independent function of the nitrate sensor NRT1.1 in Arabidopsis thaliana. J. Plant Res. 124, 425-430 (2011).

29. Kaiser, W. M. \& Brendle-Behnisch, E. Acid-base-modulation of nitrate reductase in leaf tissues. Planta 196, 1-6 (1995).

30. Puritch, G. S. \& Barker, A. V. Structure and function of tomato leaf chloroplasts during ammonium toxicity. Plant Physiol. 42, 1229-1238 (1967).

31. Carroll, A. D. et al. Ammonium assimilation and the role of $\gamma$-aminobutyric acid in $\mathrm{pH}$ homeostasis in carrot cell suspensions. Plant Physiol. 106, 513-520 (1994).

32. Hoffmann, B., Plänker, R. \& Mengel, K. Measurement of pH in the apoplast of sunflower leaves by means of fluorescence. Physiol. Plant 84, 146-153 (1992).

33. Hachiya, T. et al. Ammonium-dependent respiratory increase is dependent on the cytochrome pathway in Arabidopsis thaliana shoots. Plant Cell Environ. 33, 1888-1897 (2010).

34. Sakano, K. Revision of biochemical pH-stat: involvement of alternative pathway metabolisms. Plant Cell Physiol. 39, 467-473 (1998).

35. Schjoerring, J. K., Husted, S., Mäck, G. \& Mattsson, M. The regulation of ammonium translocation in plants. J. Exp. Bot. 53, 883-890 (2002).

36. Wallsgrove, R. M. et al. Barley mutants lacking chloroplast glutamine synthetasebiochemical and genetic analysis. Plant Physiol. 83, 155-158 (1987).

37. Betti, M. et al. Reassimilation of ammonium in Lotus japonicus. J. Exp. Bot. 65, 5557-5566 (2014).

38. Hachiya, T. \& Sakakibara, H. Interactions between nitrate and ammonium in their uptake, allocation, assimilation, and signaling in plants. J. Exp. Bot. 68, 2501-2512 (2017).

39. Marchive, C. et al. Nuclear retention of the transcription factor NLP7 orchestrates the early response to nitrate in plants. Nat. Commun. 4, 1713 (2013).

40. Okamoto, Y. et al. Shoot nitrate underlies a perception of nitrogen satiety to trigger local and systemic signaling cascades in Arabidopsis thaliana. Soil Sci. Plant Nutr. 65, 56-64 (2019).

41. Tabata, R. et al. Perception of root-derived peptides by shoot LRR-RKs mediates systemic N-demand signaling. Science 346, 343-346 (2014).

42. Hachiya, T. et al. Arabidopsis root-type ferredoxin: NADP (H) oxidoreductase 2 is involved in detoxification of nitrite in roots. Plant Cell Physiol. 57, 2440-2450 (2016).
43. Miyagi, A. et al. Principal component and hierarchical clustering analysis of metabolites in destructive weeds; polygonaceous plants. Metabolomic 6, 146-155 (2010).

44. Miyagi, A. et al. Oxalate contents in leaves of two rice cultivars grown at a free-air $\mathrm{CO}_{2}$ enrichment (FACE) site. Plant Prod. Sci. 22, 407-411 (2019).

45. Endo, M., Shimizu, H. \& Araki, T. Rapid and simple isolation of vascular, epidermal and mesophyll cells from plant leaf tissue. Nat. Protoc. 11, 1388 (2016).

46. Toyooka, K., Okamoto, T. \& Minamikawa, T. Mass transport of proform of a KDEL-tailed cysteine proteinase (SH-EP) to protein storage vacuoles by endoplasmic reticulum-derived vesicle is involved in protein mobilization in germinating seeds. J. Cell Biol. 148, 453-464 (2000).

\section{Acknowledgements}

Seeds of the A thaliana NR-null mutant and $g \ln 1 ; 2 \operatorname{gln} 1 ; 3$ were kindly provided by Dr. Nigel M. Crawford (University of California, San Diego) and by Dr. Soichi Kojima (Tohoku University, Sendai), respectively. This work was supported by the Building of Consortia for the Development of Human Resources in Science and Technology, by the Japan Society for the Promotion of Science KAKENHI Grant No. JP17K15237, by the Program for Promoting the Enhancement of Research Universities, Nagoya University by the Inamori Foundation, by the Agropolis Foundation No. 1502-405, by a Grant-in-Aid for Young Scientists from Shimane University, and by the joint research fund from Yanmar Holdings Co., Ltd. FOX lines were developed by the Plant Genome Project of RIKEN PSC, and were provided by the RIKEN BRC through the National BioResource Project of the MEXT/AMED, Japan.

\section{Author contributions}

T.H. conceived and initiated the project. T.H., A.G., and H.S. designed the experiments. T.H., J.I., M.W., M.S., K.T., A.M., M.K.-Y., D.S., and T.K. performed the experiments and data analysis. T.H., T.N., A.G., and H.S. wrote the manuscript.

\section{Competing interests}

The authors declare no competing interests.

\section{Additional information}

Supplementary information The online version contains supplementary material available at https://doi.org/10.1038/s41467-021-25238-7.

Correspondence and requests for materials should be addressed to T.H.

Peer review information Nature Communications thanks Celine Masclaux-Daubresse, Soichi Kojima and the other, anonymous, reviewer(s) for their contribution to the peer review of this work. Peer reviewer reports are available.

Reprints and permission information is available at http://www.nature.com/reprints

Publisher's note Springer Nature remains neutral with regard to jurisdictional claims in published maps and institutional affiliations.

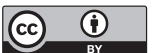

Open Access This article is licensed under a Creative Commons Attribution 4.0 International License, which permits use, sharing, adaptation, distribution and reproduction in any medium or format, as long as you give appropriate credit to the original author(s) and the source, provide a link to the Creative Commons license, and indicate if changes were made. The images or other third party material in this article are included in the article's Creative Commons license, unless indicated otherwise in a credit line to the material. If material is not included in the article's Creative Commons license and your intended use is not permitted by statutory regulation or exceeds the permitted use, you will need to obtain permission directly from the copyright holder. To view a copy of this license, visit http://creativecommons.org/ licenses/by/4.0/.

(C) The Author(s) 2021 